\title{
Approximate Controllability of Semilinear Impulsive Evolution Equations
}

\author{
Hugo Leiva \\ Departamento de Matemática, Facultad de Ciencias, Universidad de los Andes, Mérida 5101, Venezuela \\ Correspondence should be addressed to Hugo Leiva; hleiva@ula.ve
}

Received 25 August 2014; Accepted 18 October 2014

Academic Editor: Xiaofu Ji

Copyright (C) 2015 Hugo Leiva. This is an open access article distributed under the Creative Commons Attribution License, which permits unrestricted use, distribution, and reproduction in any medium, provided the original work is properly cited.

We prove the approximate controllability of the following semilinear impulsive evolution equation: $z^{\prime}=A z+B u(t)+F(t, z, u), z \in$ $Z, t \in(0, \tau], z(0)=z_{0}, z\left(t_{k}^{+}\right)=z\left(t_{k}^{-}\right)+I_{k}\left(t_{k}, z\left(t_{k}\right), u\left(t_{k}\right)\right), k=1,2,3, \ldots, p$, where $0<t_{1}<t_{2}<t_{3}<\cdots<t_{p}<\tau, Z$ and $U$ are Hilbert spaces, $u \in L^{2}(0, \tau ; U), B: U \rightarrow Z$ is a bounded linear operator, $I_{k}, F:[0, \tau] \times Z \times U \rightarrow Z$ are smooth functions, and $A: D(A) \subset Z \rightarrow Z$ is an unbounded linear operator in $Z$ which generates a strongly continuous semigroup $\{T(t)\}_{t \geq 0} \subset Z$. We suppose that $F$ is bounded and the linear system is approximately controllable on $[0, \delta]$ for all $\delta \in(0, \tau)$. Under these conditions, we prove the following statement: the semilinear impulsive evolution equation is approximately controllable on $[0, \tau]$.

\section{Introduction}

There are many practical examples of impulsive control systems: a chemical reactor system with the quantities of different chemicals serving as the states variable, a financial system with two state variables of the amount of money in a market and the saving rates of a central bank, and the growth of a population diffusing throughout its habitat which is often modeled by reaction-diffusion equation, for which much has been done under the assumption that the system parameters related to the population environment either are constant or change continuously. However, one may easily visualize situations in nature where abrupt changes such as harvesting, disasters, and instantaneous stoking may occur.

This observation motivates us to study the approximate controllability of the following semilinear impulsive evolution equation:

$$
\begin{gathered}
z^{\prime}=A z+B u(t)+F(t, z, u), \quad z \in Z, t \in(0, \tau], \\
z(0)=z_{0}, \\
z\left(t_{k}^{+}\right)=z\left(t_{k}^{-}\right)+I_{k}\left(t_{k}, z\left(t_{k}\right), u\left(t_{k}\right)\right), \quad k=1,2,3, \ldots, p,
\end{gathered}
$$

where $0<t_{1}<t_{2}<t_{3}<\cdots<t_{p}<\tau, Z$ and $U$ are Hilbert spaces, $u \in L^{2}(0, \tau ; U), B: U \rightarrow Z$ is a bounded linear operator, $I_{k}, F:[0, \tau] \times Z \times U \rightarrow Z$ are smooth functions, and $A: D(A) \subset Z \rightarrow Z$ is an unbounded linear operator in $Z$ which generates a strongly continuous semigroup $\{T(t)\}_{t \geq 0} \subset Z$.

Definition 1 (approximate controllability). System (1) is said to be approximately controllable on $[0, \tau]$ if for every $z_{0}, z_{1} \in$ $Z, \varepsilon>0$ there exists $u \in L^{2}(0, \tau ; U)$ such that the solution $z(t)$ of (1) corresponding to $u$ verifies (see Figures 1 and 2)

$$
z(0)=z_{0}, \quad\left\|z(\tau)-z_{1}\right\|_{Z}<\varepsilon .
$$

We assume the following main hypotheses:

(A) $F$ is a bounded function;

(B) linear system without impulses (8) is approximately controllable on $[\tau-\delta, \tau]$ for all $0<\delta<\tau$.

That is, the Gramian controllability operator

$$
Q_{\delta}=G_{\delta} G_{\delta}^{*}=\int_{0}^{\delta} T(t) B B^{*} T^{*}(t) d t
$$




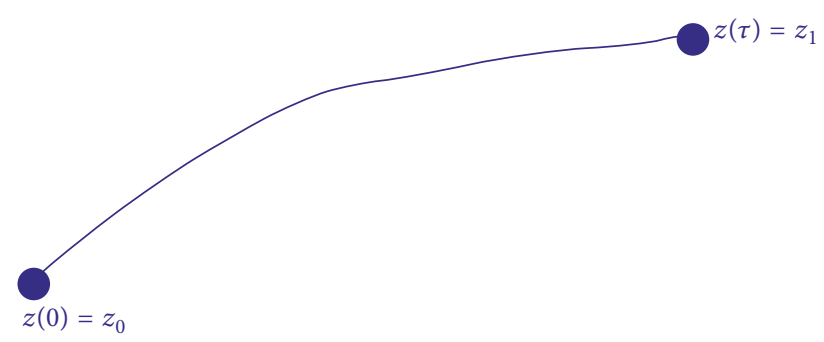

FIGURE 1

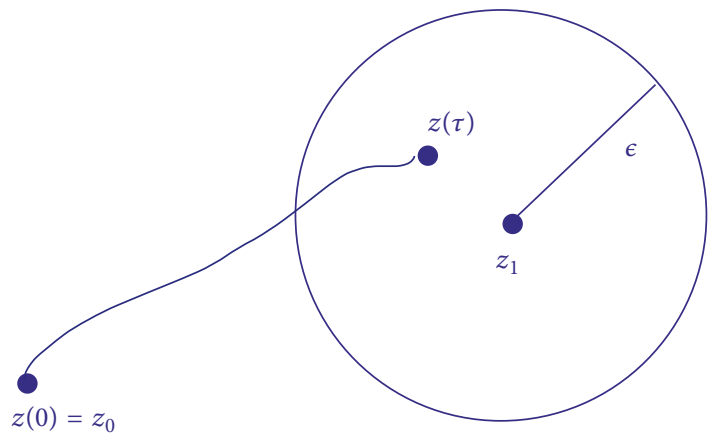

Figure 2

satisfies $Q_{\delta}>0$ for all $0<\delta<\tau$, which is equivalent, according to (13) and Lemma $3(\mathrm{c})$, to the approximate controllability of linear system (8) on $[\tau-\delta, \tau]$, for all $0<\delta<\tau$.

This paper has been motivated by the works done in Bashirov and Ghahramanlou [1], Bashirov and Jneid [2], and Bashirov et al. [3], where a new technique to prove the controllability of evolution equations without impulses is used avoiding fixed point theorems, and the work done in [4].

The controllability of impulsive evolution equations has been studied recently by several authors, but most of them study the exact controllability only; it is worth mentioning that Chalishajar [5] studied the exact controllability of impulsive partial neutral functional differential equations with infinite delay, Radhakrishnan and Balachandran [6] studied the exact controllability of semilinear impulsive integrodifferential evolution systems with nonlocal conditions, and Selvi and Mallika Arjunan [7] studied the exact controllability for impulsive differential systems with finite delay. To our knowledge, there are a few works on approximate controllability of impulsive semilinear evolution equations worth mentioning: Chen and Li [8] studied the approximate controllability of impulsive differential equations with nonlocal conditions, using measure of noncompactness and Monch fixed point theorem and assuming that the nonlinear term $f(t, z)$ does not depend on the control variable, and Leiva and Merentes in [4] studied the approximate controllability of the semilinear impulsive heat equation using the fact that the semigroup generated by $\Delta$ is compact.

In this paper, we are not assuming the compactness of the semigroup $\{T(t)\}_{t \geq 0}$ generated by the unbounded operator $A$; when this semigroup is compact we can consider weaker condition on the nonlinear perturbation $F$ and in the linear part of the system without impulses. Specifically, we can assume the following hypotheses:

(a)

$$
\begin{gathered}
\|F(t, z, u)\|_{Z} \leq a_{0}\|z\|_{Z}^{\alpha_{0}}+b_{0}\|u\|_{Z}^{\beta_{0}}+c_{0}, \\
\left\|I_{k}(t, z, u)\right\|_{Z} \leq a_{k}\|z\|_{Z}^{\alpha_{k}}+b_{k}\|u\|_{Z}^{\beta_{k}}+c_{k},
\end{gathered}
$$

$$
k=1,2,3, \ldots, p,
$$

with $1 / 2 \leq \alpha_{k}<1,1 / 2 \leq \beta_{k}<1, k=0,1,2,3, \ldots, p ;$

(b) the linear system is approximately controllable only on $[0, \tau]$.

This case is similar to the semilinear impulsive heat equations studied in [4], where the authors use conditions (a) and (b), the compactness of the semigroup generated by the Laplacian operator $\Delta$, and Rothe's fixed point theorem to prove the approximate controllability of the system on $[0, \tau]$.

When it comes to the wave equation, the situation is totally different; the semigroup generated by the linear part is not compact; it is in fact a group, which can never be compact. Furthermore, if the control acts on a portion $\omega$ of the domain $\Omega$ for the spatial variable, then the system is approximately controllable only on $[0, \tau]$ for $\tau \geq 2$, which was proved in [9], where the following system governed by the wave equations was studied:

$$
\begin{gathered}
y_{t t}=\Delta y+1_{\omega} u(t, x), \quad \text { on }(0, \tau) \times \Omega, \\
y=0, \quad \text { on }(0, \tau) \times \partial \Omega, \\
y(0, x)=y_{0}(x), \quad y_{t}(0, x)=y_{1}(x), \quad \text { in } \Omega,
\end{gathered}
$$

where $\Omega$ is a bounded domain in $\mathbb{R}^{n}, \omega$ is an open nonempty subset of $\Omega, 1_{\omega}$ denotes the characteristic function of the set $\omega$, the distributed control $u \in L_{2}\left([0, \tau] ; L_{2}(\Omega)\right)$, and $y_{0} \in$ $H^{2}(\Omega) \cap H_{0}^{1}, y_{1} \in L_{2}(\Omega)$.

However, if the control acts on the whole domain $\Omega$, it was proved in [10] that the system is controllable $[0, \tau]$, for all $\tau>0$. More specifically, the authors studied the following system:

$$
\begin{gathered}
y_{t t}=\Delta y+u(t, x), \quad \text { on }(0, \tau) \times \Omega, \\
y=0, \quad \text { on }(0, \tau) \times \partial \Omega, \\
y(0, x)=y_{0}(x), \quad y_{t}(0, x)=y_{1}(x), \quad \text { in } \Omega,
\end{gathered}
$$

where $\Omega$ is a bounded domain in $\mathbb{R}^{n}$, the distributed control $u \in L_{2}\left([0, \tau] ; L_{2}(\Omega)\right)$, and $y_{0} \in H^{2}(\Omega) \cap H_{0}^{1}, y_{1} \in L_{2}(\Omega)$.

To justify the use of this new technique [1], in this paper, we consider as an application the semilinear impulsive wave 
equation with controls acting on the whole domain $\Omega$, so that hypotheses (A) and (B) hold:

$$
\begin{gathered}
y_{t t}=\Delta y+u(t, x)+f\left(t, y, y_{t}, u(t)\right), \quad \text { on }(0, \tau) \times \Omega, \\
y=0, \quad \text { on }(0, \tau) \times \partial \Omega, \\
y(0, x)=y_{0}(x), \quad y_{t}(0, x)=y_{1}(x), \quad \text { in } \Omega, \\
y_{t}\left(t_{k}^{+}, x\right)=y_{t}\left(t_{k}^{-}, x\right) \\
+I_{k}\left(t, y\left(t_{k}, x\right), y_{t}\left(t_{k}, x\right), u\left(t_{k}, x\right)\right), \quad x \in \Omega,
\end{gathered}
$$

where $0<t_{1}<t_{2}<t_{3}<\cdots<t_{p}<\tau$, $\Omega$ is a bounded domain in $\mathbb{R}^{n}$, the distributed control $u \in L_{2}\left([0, \tau] ; L_{2}(\Omega)\right)$, $y_{0} \in H^{2}(\Omega) \cap H_{0}^{1}, y_{1} \in L_{2}(\Omega)$, and $I_{k}, f$ are smooth functions with $f$ being bounded.

\section{Controllability of the Linear Equation without Impulses}

In this section we will present some characterization of the approximate controllability of the corresponding linear equations without impulses. To this end, we note that for all $z_{0} \in Z$ and $u \in L_{2}(0, \tau ; U)$ the initial value problem

$$
\begin{gathered}
z^{\prime}=A z+B u(t), \quad z \in Z, \\
z\left(t_{0}\right)=z_{0}
\end{gathered}
$$

admits only one mild solution given by

$$
\begin{aligned}
z(t)= & z\left(t, t_{0}, z_{0}, u\right) \\
= & T(t) z_{0} \\
& +\int_{t_{0}}^{t} T(t-s) B u(s) d s, \quad t \in\left[t_{0}, \tau\right], 0 \leq t_{0} \leq \tau .
\end{aligned}
$$

Definition 2. For system (8), one defines the following concept: the controllability maps $G_{\tau \delta}: L^{2}(\tau-\delta, \tau ; U) \rightarrow Z$, $G_{\delta}: L^{2}(0, \delta ; U) \rightarrow Z$, defined by

$$
\begin{gathered}
G_{\tau \delta} u=\int_{\tau-\delta}^{\tau} T(\tau-s) B u(s) d s, \quad u \in L^{2}(\tau-\delta, \tau ; U), \\
G_{\delta} v=\int_{0}^{\delta} T(s) B v(s) d s, \quad v \in L^{2}(0, \delta ; U),
\end{gathered}
$$

satisfy the following relation:

$$
\begin{aligned}
G_{\tau \delta} u & =\int_{\tau-\delta}^{\tau} T(\tau-s) B u(s) d s \\
& =\int_{0}^{\delta} T(s) B u(\tau-s) d s \\
& =G_{\delta} u(\tau-\cdot) .
\end{aligned}
$$

The adjoints of these operators $G_{\tau \delta}^{*}: Z \rightarrow L^{2}(\tau-\delta, \tau ; U)$, $G_{\delta}^{*}: Z \rightarrow L^{2}(0, \delta ; U)$ are given by

$$
\begin{gathered}
\left(G_{\tau \delta}^{*} z\right)(t)=B^{*} T^{*}(\tau-t) z, \quad t \in[\tau-\delta, \tau], \\
\left(G_{\delta}^{*} x\right)(t)=B^{*} T^{*}(t) z, \quad t \in[0, \delta] .
\end{gathered}
$$

The Gramian controllability operators are given by (3) and

$$
Q_{\tau \delta}=G_{\tau \delta} G_{\tau \delta}^{*}=\int_{\tau-\delta}^{\tau} T(\tau-t) B B^{*} T^{*}(\tau-t) d t=Q_{\delta} .
$$

The following lemma holds in general for a linear bounded operator $G: W \rightarrow Z$ between Hilbert spaces $W$ and $Z$ (see $[4,11,12])$.

Lemma 3. The following statements are equivalent to the approximate controllability of the linear system (8) on $[\tau-\delta, \tau]$.

(a) $\overline{\text { Range }\left(G_{\tau \delta}\right)}=Z$.

(b) $\operatorname{Ker}\left(G_{\tau \delta}^{*}\right)=\{0\}$.

(c) $\left\langle Q_{\tau \delta} z, z\right\rangle>0, z \neq 0$ in $Z$.

(d) $\lim _{\alpha \rightarrow 0^{+}} \alpha\left(\alpha I+Q_{\tau \delta}\right)^{-1} z=0$.

(e) For all $z \in Z$, one has $G_{\tau \delta} u_{\alpha}=z-\alpha\left(\alpha I+Q_{T \delta}\right)^{-1} z$, where

$$
u_{\alpha}=G_{\tau \delta}^{*}\left(\alpha I+Q_{\tau \delta}\right)^{-1} z, \quad \alpha \in(0,1] .
$$

So $\lim _{\alpha \rightarrow 0} G_{\tau \delta} u_{\alpha}=z$ and the error $E_{\tau \delta} z$ of this approximation is given by the formula

$$
E_{\tau \delta} z=\alpha\left(\alpha I+Q_{\tau \delta}\right)^{-1} z, \quad \alpha \in(0,1] .
$$

(f) Moreover, if one considers for each $v \in L^{2}(\tau-\delta, \tau$; $U)$ the sequence of controls given by

$$
\begin{aligned}
u_{\alpha}= & G_{\tau \delta}^{*}\left(\alpha I+Q_{\tau \delta}\right)^{-1} z \\
& +\left(v-G_{\tau \delta}^{*}\left(\alpha I+Q_{\tau \delta}\right)^{-1} G_{\tau \delta} v\right), \quad \alpha \in(0,1],
\end{aligned}
$$

one gets that

$$
\begin{gathered}
G_{\tau \delta} u_{\alpha}=z-\alpha\left(\alpha I+Q_{T \delta}\right)^{-1}\left(z+G_{\tau \delta} v\right), \\
\lim _{\alpha \rightarrow 0} G_{\tau \delta} u_{\alpha}=z,
\end{gathered}
$$

with the error $E_{\tau \delta} z$ of this approximation given by the formula

$$
E_{\tau \delta} z=\alpha\left(\alpha I+Q_{\tau \delta}\right)^{-1}\left(z+G_{\tau \delta} v\right), \quad \alpha \in(0,1] .
$$

Remark 4. The foregoing lemma implies that the family of linear operators $\Gamma_{\alpha \tau \delta}: Z \rightarrow W$, defined for $0<\alpha \leq 1$ by

$$
\Gamma_{\alpha \tau \delta} z=G_{\tau \delta}^{*}\left(\alpha I+Q_{\tau \delta}\right)^{-1} z,
$$

is an approximate inverse for the right of the operator $W$, in the sense that

$$
\lim _{\alpha \rightarrow 0} G_{\tau \delta} \Gamma_{\alpha \tau \delta}=I
$$

in the strong topology. 
Lemma 5. $Q_{\delta}>0$ if and only if linear system (8) is controllable on $[\tau-\delta, \tau]$. Moreover, given an initial state $y_{0}$ and a final state $z_{1}$, one can find a sequence of controls $\left\{u_{\alpha}^{\delta}\right\}_{0<\alpha \leq 1} \subset L^{2}(\tau-$ $\delta, \tau ; U)$ :

$$
u_{\alpha}=G_{\tau \delta}^{*}\left(\alpha I+G_{\tau \delta} G_{\tau \delta}^{*}\right)^{-1}\left(z_{1}-T(\tau) y_{0}\right), \quad \alpha \in(0,1],
$$

such that the solutions $y(t)=y\left(t, \tau-\delta, y_{0}, u_{\alpha}^{\delta}\right)$ of the initial value problem

$$
\begin{gathered}
y^{\prime}=A y+B u_{\alpha}(t), \quad y \in Z, t>0 \\
y(\tau-\delta)=y_{0}
\end{gathered}
$$

satisfy

$$
\lim _{\alpha \rightarrow 0^{+}} y\left(\tau, \tau-\delta, y_{0}, u_{\alpha}\right)=z_{1}
$$

that is,

$$
\begin{aligned}
& \lim _{\alpha \rightarrow 0^{+}} y(\tau) \\
& \quad=\lim _{\alpha \rightarrow 0^{+}}\left\{T(\delta) y_{0}+\int_{\tau-\delta}^{\tau} T(\tau-s) B u_{\alpha}(s) d s\right\}=z_{1} .
\end{aligned}
$$

\section{Controllability of the Semilinear Impulsive System}

In this section, we will prove the main result of this paper: the approximate controllability of the semilinear impulsive evolution equation given by (1). To this end, for all $z_{0} \in Z$ and $u \in L^{2}(0, \tau ; U)$, the initial value problem

$$
\begin{gathered}
z^{\prime}=A z+B u+F(t, z, u), \quad t \in(0, \tau], t \neq t_{k}, z \in Z, \\
z(0)=z_{0}, \\
z\left(t_{k}^{+}\right)=z\left(t_{k}^{-}\right)+I_{k}\left(t, z\left(t_{k}\right), u\left(t_{k}\right)\right), \quad k=1,2,3, \ldots, p,
\end{gathered}
$$

admits only one mild solution given by

$$
\begin{aligned}
z_{u}(t)= & T(t) z_{0}+\int_{0}^{t} T(t-s) B u(s) d s \\
& +\int_{0}^{t} T(t-s) F\left(s, z_{u}(s), u(s)\right) d s \\
& +\sum_{0<t_{k}<t} T\left(t-t_{k}\right) I_{k}\left(t_{k}, z\left(t_{k}\right), u\left(t_{k}\right)\right), \quad t \in[0, \tau] .
\end{aligned}
$$

Now, we are ready to present and prove the main result of this paper, which is the approximate controllability of semilinear impulsive equation (1).

Theorem 6. Under conditions (A) and (B), semilinear impulsive system (1) is approximately controllable on $[0, \tau]$.
Proof. Given an initial state $z_{0}$, a final state $z_{1}$, and $\epsilon>0$, we want to find a control $u_{\alpha}^{\delta} \in L^{2}(0, \tau ; U)$ steering the system from $z_{0}$ to an $\epsilon$-neighborhood of $z_{1}$ on time $\tau$. Specifically,

$$
\begin{aligned}
\lim _{\alpha \rightarrow 0}\{ & T(\tau) z_{0}+\int_{0}^{\tau} T(\tau-s) B u_{\alpha}^{\delta}(s) d s \\
& +\int_{0}^{\tau} T(\tau-s) F\left(s, z_{\alpha}(s), u_{\alpha}^{\delta}(s)\right) d s \\
& \left.+\sum_{0<t_{k}<\tau} T\left(\tau-t_{k}\right) I_{k}\left(z_{\alpha}\left(t_{k}\right), u_{\alpha}^{\delta}\left(t_{k}\right)\right)\right\}=z_{1} .
\end{aligned}
$$

Consider any $u \in L^{2}(0, \tau ; U)$ and the corresponding solution $z(t)=z\left(t, 0, z_{0}, u\right)$ of initial value problem (25). For $\alpha \epsilon$ $(0,1]$, we define the control $u_{\alpha}^{\delta} \in L^{2}(0, \tau ; U)$ as follows:

$$
u_{\alpha}^{\delta}(t)= \begin{cases}u(t), & \text { if } 0 \leq t \leq \tau-\delta \\ u_{\alpha}(t), & \text { if } \tau-\delta<t \leq \tau\end{cases}
$$

where

$$
\begin{array}{r}
u_{\alpha}(t)=B^{*} T^{*}(\tau-t)\left(\alpha I+G_{\tau \delta} G_{\tau \delta}^{*}\right)^{-1}\left(z_{1}-T(\delta) z(\tau-\delta)\right), \\
\tau-\delta<t \leq \tau .
\end{array}
$$

Now, assume that $0<\delta<\tau-t_{p}$. Then the corresponding solution $z_{\alpha}^{\delta}(t)=z\left(t, 0, z_{0}, u_{\alpha}^{\delta}\right)$ of initial value problem (25) at time $\tau$ can be written as follows:

$$
\begin{aligned}
& z_{\alpha}^{\delta}(\tau)=T(\tau) z_{0}+\int_{0}^{\tau} T(\tau-s) B u_{\alpha}^{\delta}(s) d s \\
& +\int_{0}^{\tau} T(\tau-s) F\left(s, z_{\alpha}^{\delta}(s), u_{\alpha}^{\delta}(s)\right) d s \\
& +\sum_{0<t_{k}<\tau} T\left(\tau-t_{k}\right) I_{k}^{e}\left(z_{\alpha}^{\delta}\left(t_{k}\right), u_{\alpha}^{\delta}\left(t_{k}\right)\right) \\
& =T(\delta)\left\{T(\tau-\delta) z_{0}+\int_{0}^{\tau-\delta} T(\tau-\delta-s) B u_{\alpha}^{\delta}(s) d s\right. \\
& +\int_{0}^{\tau-\delta} T(\tau-\delta-s) F\left(s, z_{\alpha}^{\delta}(s), u_{\alpha}^{\delta}(s)\right) d s \\
& \left.+\sum_{0<t_{k}<\tau-\delta} T\left(\tau-\delta-t_{k}\right) I_{k}^{e}\left(z_{\alpha}^{\delta}\left(t_{k}\right), u_{\alpha}^{\delta}\left(t_{k}\right)\right)\right\} \\
& +\int_{\tau-\delta}^{\tau} T(\tau-s) B u_{\alpha}^{\delta}(s) d s \\
& +\int_{\tau-\delta}^{\tau} T(\tau-s) F\left(s, z_{\alpha}^{\delta}(s), u_{\alpha}^{\delta}(s)\right) d s \\
& =T(\delta) z(\tau-\delta)+\int_{\tau-\delta}^{\tau} T(\tau-s) B u_{\alpha}(s) d s \\
& +\int_{\tau-\delta}^{\tau} T(\tau-s) F\left(s, z_{\alpha}^{\delta}(s), u_{\alpha}(s)\right) d s .
\end{aligned}
$$


So,

$$
\begin{aligned}
z_{\alpha}^{\delta}(\tau)= & T(\delta) z(\tau-\delta)+\int_{\tau-\delta}^{\tau} T(\tau-s) B u_{\alpha}(s) d s \\
& +\int_{\tau-\delta}^{\tau} T(\tau-s) F\left(s, z_{\alpha}^{\delta}(s), u_{\alpha}(s)\right) d s .
\end{aligned}
$$

The corresponding solution $y_{\alpha}^{\delta}(t)=y\left(t, \tau-\delta, z(\tau-\delta), u_{\alpha}\right)$ of initial value problem (22) at time $\tau$ is given by

$$
y_{\alpha}^{\delta}(\tau)=T(\delta) z(\tau-\delta)+\int_{\tau-\delta}^{\tau} T(\tau-s) B u_{\alpha}(s) d s .
$$

Therefore,

$$
\left\|z_{\alpha}^{\delta}(\tau)-y_{\alpha}^{\delta}(\tau)\right\| \leq \int_{\tau-\delta}^{\tau}\|T(\tau-s)\|\left\|F\left(s, z_{\alpha}^{\delta}(s), u_{\alpha}(s)\right)\right\| d s .
$$

Then, putting $M=\sup _{0 \leq t \leq \tau}\|T(t)\|$ and $K=\sup _{[0, \tau] \times Z \times U} \| F(t$, $z, u) \|$, we obtain the following estimate:

$$
\left\|z_{\alpha}^{\delta}(\tau)-y_{\alpha}^{\delta}(\tau)\right\| \leq M K \delta
$$

Hence,

$$
\left\|z_{\alpha}^{\delta}(\tau)-z_{1}\right\| \leq M K \delta+\left\|y_{\alpha}^{\delta}(\tau)-z_{1}\right\| .
$$

From Lemma 5, there exists $\alpha>0$ such that

$$
\left\|y_{\alpha}^{\delta}(\tau)-z_{1}\right\| \leq \frac{\epsilon}{2} .
$$

So, taking $\delta<\min \left\{\tau-t_{p}, \epsilon / 2 M K\right\}$, we obtain for the corresponding control $u_{\alpha}^{\delta}$ that

$$
\left\|z_{\alpha}^{\delta}(\tau)-z_{1}\right\| \leq \epsilon .
$$

Geometrically, the proof goes as shown in Figure 3.

This completes the proof of the theorem.

\section{Applications}

As an application, we will prove the approximate controllability of the following control system governed by the semilinear impulsive wave equation:

$$
\begin{array}{r}
y_{t t}=\Delta y+u(t, x)+f\left(t, y, y_{t}, u(t)\right), \quad \text { on }(0, \tau) \times \Omega, \\
y=0, \quad \text { on }(0, \tau) \times \partial \Omega, \\
y(0, x)=y_{0}(x), \quad y_{t}(0, x)=y_{1}(x), \quad \text { in } \Omega, \\
y_{t}\left(t_{k}^{+}, x\right)=y_{t}\left(t_{k}^{-}, x\right)+I_{k}\left(t, y\left(t_{k}, x\right), y_{t}\left(t_{k}, x\right), u\left(t_{k}, x\right)\right), \\
x \in \Omega,
\end{array}
$$

where $\Omega$ is a bounded domain in $\mathbb{R}^{n}$, the distributed control $u \in L_{2}\left([0, \tau] ; L_{2}(\Omega)\right), y_{0} \in H^{2}(\Omega) \cap H_{0}^{1}, y_{1} \in L_{2}(\Omega)$, and $I_{k}, f$ are smooth functions with $f$ being bounded.

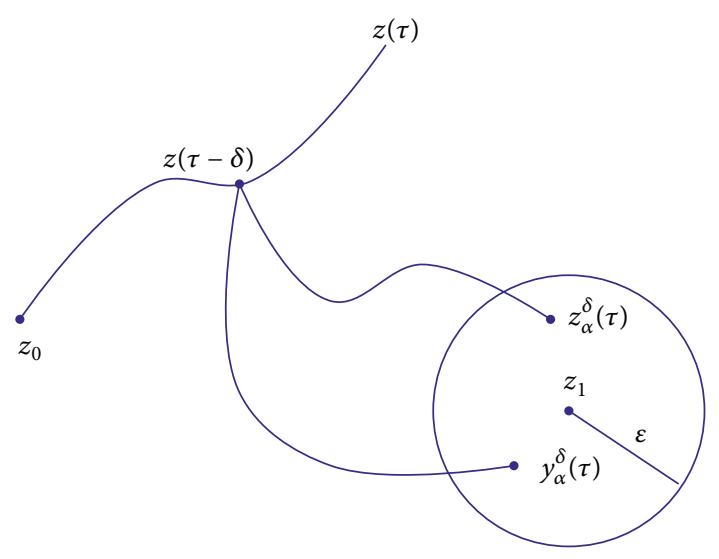

FIGURE 3

4.1. Abstract Formulation of the Problem. In this part, we will choose the space where this problem will be set up as an abstract control system in a Hilbert space. Let $X=L_{2}(\Omega)=$ $L_{2}(\Omega, \mathbb{R})$ and consider the linear unbounded operator $A$ : $D(A) \subset X \rightarrow X$ defined by $A \phi=-\Delta \phi$, where

$$
D(A)=H^{2}(\Omega, \mathbb{R}) \cap H_{0}^{1}(\Omega, \mathbb{R}) .
$$

Then the eigenvalues $\lambda_{j}$ of $A$ have finite multiplicity $\gamma_{j}$ equal to the dimension of the corresponding eigenspace and $0<$ $\lambda_{1}<\lambda_{2}<\cdots<\lambda_{n} \rightarrow \infty$. Moreover, consider the following.

(a) There exists a complete orthonormal set $\left\{\phi_{j, k}\right\}$ of eigenvectors of $A$.

(b) For all $x \in D(A)$, we have

$$
A x=\sum_{j=1}^{\infty} \lambda_{j} \sum_{k=1}^{\gamma_{j}}\left\langle x, \phi_{j, k}\right\rangle \phi_{j, k}=\sum_{j=1}^{\infty} \lambda_{j} E_{j} x,
$$

where $\langle\cdot, \cdot\rangle$ is the usual inner product in $L^{2}$ and

$$
E_{j} x=\sum_{k=1}^{\gamma_{j}}\left\langle x, \phi_{j, k}\right\rangle \phi_{j, k},
$$

which means the set $\left\{E_{j}\right\}_{j=1}^{\infty}$ is a complete family of orthogonal projections in $X$ and $x=\sum_{j=1}^{\infty} E_{j} x, x \in X$.

(c) $-A$ generates an analytic semigroup $\left\{e^{-A t}\right\}$ given by

$$
e^{-A t} x=\sum_{j=1}^{\infty} e^{-\lambda_{j} t} E_{j} x
$$

(d) The fractional powered spaces $X^{r}$ are given by

$$
X^{r}=D\left(A^{r}\right)=\left\{x \in X: \sum_{n=1}^{\infty} \lambda_{n}^{2 r}\left\|E_{n} x\right\|^{2}<\infty\right\}, \quad r \geq 0,
$$


with the norm

$$
\begin{gathered}
\|x\|_{r}=\left\|A^{r} x\right\|=\left\{\sum_{n=1}^{\infty} \lambda_{n}^{2 r}\left\|E_{n} x\right\|^{2}\right\}^{1 / 2}, \quad x \in X^{r}, \\
A^{r} x=\sum_{n=1}^{\infty} \lambda_{n}^{r} E_{n} x .
\end{gathered}
$$

Also, for $r \geq 0$, we define $Z_{r}=X^{r} \times X$, which is a Hilbert space endowed with the norm given by

$$
\left\|\left[\begin{array}{l}
y \\
v
\end{array}\right]\right\|_{Z_{r}}=\sqrt{\|y\|_{r}^{2}+\|v\|^{2}} .
$$

Then, (1) can be written as abstract second order ordinary differential equations in $Z_{1 / 2}$ as follows:

$$
\begin{array}{r}
y^{\prime \prime}=-A y+u+f^{e}\left(t, y, y^{\prime}, u\right), \quad t \in(0, \tau], t \neq t_{k}, \\
y(0)=y_{0}, \quad y^{\prime}(0)=y_{1}, \\
y^{\prime}\left(t_{k}^{+}\right)=y^{\prime}\left(t_{k}^{-}\right)+I_{k}^{e}\left(t_{k}, y\left(t_{k}\right), y^{\prime}\left(t_{k}\right), u\left(t_{k}\right)\right), \\
k=1,2,3, \ldots, p,
\end{array}
$$

where $I_{k}^{e}, f^{e}:[0, \tau] \times Z_{1 / 2} \times U \rightarrow Z_{1 / 2}$ are defined by

$$
\begin{array}{r}
I_{k}^{e}(t, y, v, u)(x)=I_{k}(t, y(x), v(x), u(x)), \\
f^{e}(t, z, u)(x)=f(t, y(x), v(x), u(x)), \\
\forall x \in \Omega, \quad k=1,2, \ldots, p .
\end{array}
$$

With the change of variables $y^{\prime}=v$, we can write second order equation (46) as a first order system of ordinary differential equations in the Hilbert space $Z_{1 / 2}=X^{1 / 2} \times X$ as follows:

$$
\begin{gathered}
z^{\prime}=\mathscr{A} z+B u+F(t, z, u(t)), \quad z \in Z_{1 / 2}, t \in(0, \tau], t \neq t_{k}, \\
z(0)=z_{0}, \\
z\left(t_{k}^{+}\right)=z\left(t_{k}^{-}\right)+J_{k}\left(t_{k}, z\left(t_{k}\right), u\left(t_{k}\right)\right), \quad k=1,2,3, \ldots, p,
\end{gathered}
$$

where $u \in L_{2}([0, \tau] ; U), U=X=L_{2}(\Omega)$,

$$
z=\left[\begin{array}{l}
y \\
v
\end{array}\right], \quad B=\left[\begin{array}{l}
0 \\
I
\end{array}\right], \quad \mathscr{A}=\left[\begin{array}{cc}
0 & I_{X} \\
-A & 0
\end{array}\right]
$$

is an unbounded linear operator with domain $D(\mathscr{A})=$ $D(A) \times D\left(A^{1 / 2}\right)$, and $J_{k}, F:[0, \tau] \times Z_{1 / 2} \times U \rightarrow Z_{1 / 2}$ are defined by

$$
\begin{aligned}
& F(t, z, u)=\left[\begin{array}{c}
0 \\
f^{e}(t, y, v, u)
\end{array}\right], \\
& J_{k}(t, z, u)=\left[\begin{array}{c}
0 \\
I_{k}^{e}(t, w, v, u)
\end{array}\right] .
\end{aligned}
$$

It is well known that the operator $\mathscr{A}$ generates a strongly continuous group $\{T(t)\}_{t \geq 0}$ in the space $Z=Z_{1 / 2}=X^{1 / 2} \times X$ (see [13]). The following representation for this group can be found in [9] as Theorem 2.2.
Proposition 7. The group $\{T(t)\}_{t \geq 0}$ generated by the operator $A$ has the following representation:

$$
T(t) z=\sum_{n j=1}^{\infty} e^{A_{j} t} P_{j} z, \quad z \in Z_{1 / 2}, \quad t \geq 0,
$$

where $\left\{P_{j}\right\}_{j \geq 0}$ is a complete family of orthogonal projections in the Hilbert space $Z_{1 / 2}$ given by

$$
\begin{gathered}
P_{j}=\left[\begin{array}{cc}
E_{j} & 0 \\
0 & E_{j}
\end{array}\right], \quad j \geq 1, \\
A_{j}=R_{j} P_{j}, \quad R_{j}=\left[\begin{array}{cc}
0 & 1 \\
-\lambda_{j} & 0
\end{array}\right], \quad j \geq 1 .
\end{gathered}
$$

4.2. Approximate Controllability. Now, we are ready to formulate and prove the main result of this section, which is the approximate of the semilinear impulsive wave equation with bounded nonlinear perturbation.

Theorem 8. Semilinear impulsive wave equation (38) is approximately controllable on $[0, \tau]$.

Proof. From [10], we know that the corresponding linear system without impulses

$$
\begin{gathered}
z^{\prime}=\mathscr{A} z+B u, \quad z \in Z_{1 / 2}, t \in(0, \tau] \\
z(0)=z_{0}
\end{gathered}
$$

is exactly controllable on $[0, \delta]$, for all $0<\delta<\tau$. On the other hand, since $f$ is bounded, there exists $M>0$ such that $\sup _{[0, \tau] \times \mathbb{R} \times \mathbb{R} \times \mathbb{R}}\|f(t, y, v, u)\| \leq M$. Therefore, we obtain

$$
\begin{aligned}
& \|F(t, z, u)\|_{Z_{1 / 2}} \\
& \quad=\left\{\int_{\Omega}|f(t, y(x), v(x), u(x))|^{2} d x\right\}^{1 / 2} \leq \sqrt{\mu(\Omega)} M,
\end{aligned}
$$

for all $(t, z, u) \in[0, \tau] \times Z_{1 / 2} \times U$ with $\mu(\Omega)$ the Lebesgue measure of $\Omega$. Hence hypotheses (A) and (B) in Theorem 6 are satisfied and we get the result.

\section{Final Remark}

This technique can be applied to those systems where the linear part does not generate a compact semigroup and is controllable on any $[0, \delta]$ for $\delta>0$ and the nonlinear perturbation is bounded. Example of such systems is the following controlled thermoelastic plate equation whose linear part was studied in [10]:

$$
\begin{array}{r}
y_{t t}+\Delta^{2} y+\alpha \Delta \theta=u_{1}(t, x)+f_{1}\left(t, y, y_{t}, \theta, u(t)\right), \\
\text { on }(0, \tau) \times \Omega,
\end{array}
$$




$$
\begin{gathered}
\theta_{t}-\beta \Delta \theta-\alpha \Delta y_{t}=u_{2}(t, x)+f_{2}\left(t, y, y_{t}, \theta, u(t)\right), \\
\text { on }(0, \tau) \times \Omega, \\
\theta=y=\Delta y=0, \quad \text { on }(0, \tau) \times \partial \Omega, \\
y_{t}\left(t_{k}^{+}, x\right)=y_{t}\left(t_{k}^{-}, x\right)+I_{k}^{1}\left(t, y\left(t_{k}, x\right), y_{t}\left(t_{k}, x\right), u\left(t_{k}, x\right)\right), \\
x \in \Omega, \\
\theta\left(t_{k}^{+}, x\right)=\theta\left(t_{k}^{-}, x\right)+I_{k}^{2}\left(t_{k}, \theta\left(t_{k}, x\right), u\left(t_{k}, x\right)\right), \quad x \in \Omega,
\end{gathered}
$$

in the space $Z=X^{1} \times X \times X$, where $\Omega$ is a bounded domain in $\mathbb{R}^{n}$, the distributed controls $u_{1}, u_{2} \in L_{2}\left([0, \tau] ; L_{2}(\Omega)\right)$, and $I_{k}^{i}, f_{i}$ are smooth functions with $f_{i}, i=1,2$, being bounded.

\section{Conflict of Interests}

The author declares that there is no conflict of interests regarding the publication of this paper.

\section{Acknowledgments}

This work has been supported by CDCHT-ULA-C-1796-1205-AA and BCV.

\section{References}

[1] A. E. Bashirov and N. Ghahramanlou, "On partial approximate controllability of semilinear systems," Cogent Engineering, vol. 1, no. 1, 2014.

[2] A. E. Bashirov and M. Jneid, "On partial complete controllability of semilinear systems," Abstract and Applied Analysis, vol. 2013, Article ID 521052, 8 pages, 2013.

[3] A. E. Bashirov, N. Mahmudov, N. Şemı, and H. Etıkan, "Partial controllability concepts," International Journal of Control, vol. 80, no. 1, pp. 1-7, 2007.

[4] H. Leiva and N. Merentes, "Approximate controllability of the impul sive semilinear heat equation," to appear in Journal of Mathematics and Applications.

[5] D. N. Chalishajar, "Controllability of impulsive partial neutral functional differential equation with infinite delay," International Journal of Mathematical Analysis, vol. 5, no. 5-8, pp. 369380, 2011.

[6] B. Radhakrishnan and K. Balachandran, "Controllability results for semilinear impulsive integrodifferential evolution systems with nonlocal conditions," Journal of Control Theory and Applications, vol. 10, no. 1, pp. 28-34, 2012.

[7] S. Selvi and M. Mallika Arjunan, "Controllability results for impulsive differential systems with finite delay," Journal of Nonlinear Science and Its Applications, vol. 5, no. 3, pp. 206-219, 2012.

[8] L. Chen and G. Li, "Approximate controllability of impulsive differential equations with nonlocal conditions," International Journal of Nonlinear Science, vol. 10, no. 4, pp. 438-446, 2010.

[9] H. Leiva and N. Merentes, "Controllability of second-order equations in $L^{2}(\Omega)$," Mathematical Problems in Engineering, vol. 2010, Article ID 147195, 11 pages, 2010.
[10] H. Lárez, H. Leiva, and J. Uzcátegui, “Controllability of block diagonal systems and applications," International Journal of Systems, Control and Communications, vol. 3, no. 1, 2011.

[11] R. F. Curtain and A. J. Pritchard, Infinite Dimensional Linear Systems, vol. 8 of Lecture Notes in Control and Information Sciences, Springer, Berlin, Germany, 1978.

[12] R. F. Curtain and H. J. Zwart, An Introduction to Infinite Dimensional Linear Systems Theory, vol. 21 of Text in Applied Mathematics, Springer, New York, NY, USA, 1995.

[13] S. P. Chen and R. Triggiani, "Proof of extensions of two conjectures on structural damping for elastic systems," Pacific Journal of Mathematics, vol. 136, no. 1, pp. 15-55, 1989. 


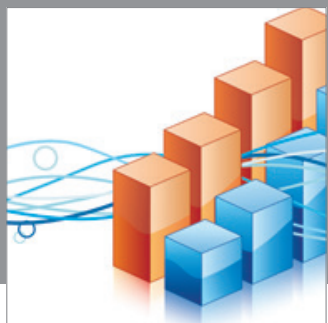

Advances in

Operations Research

mansans

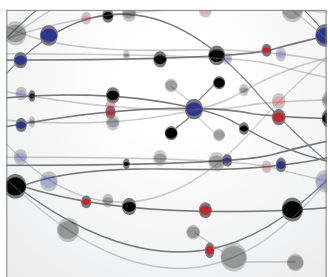

The Scientific World Journal
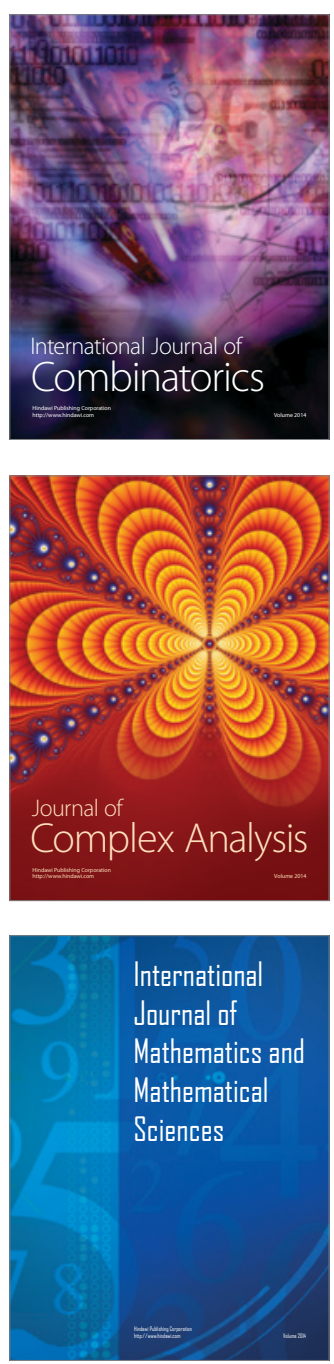
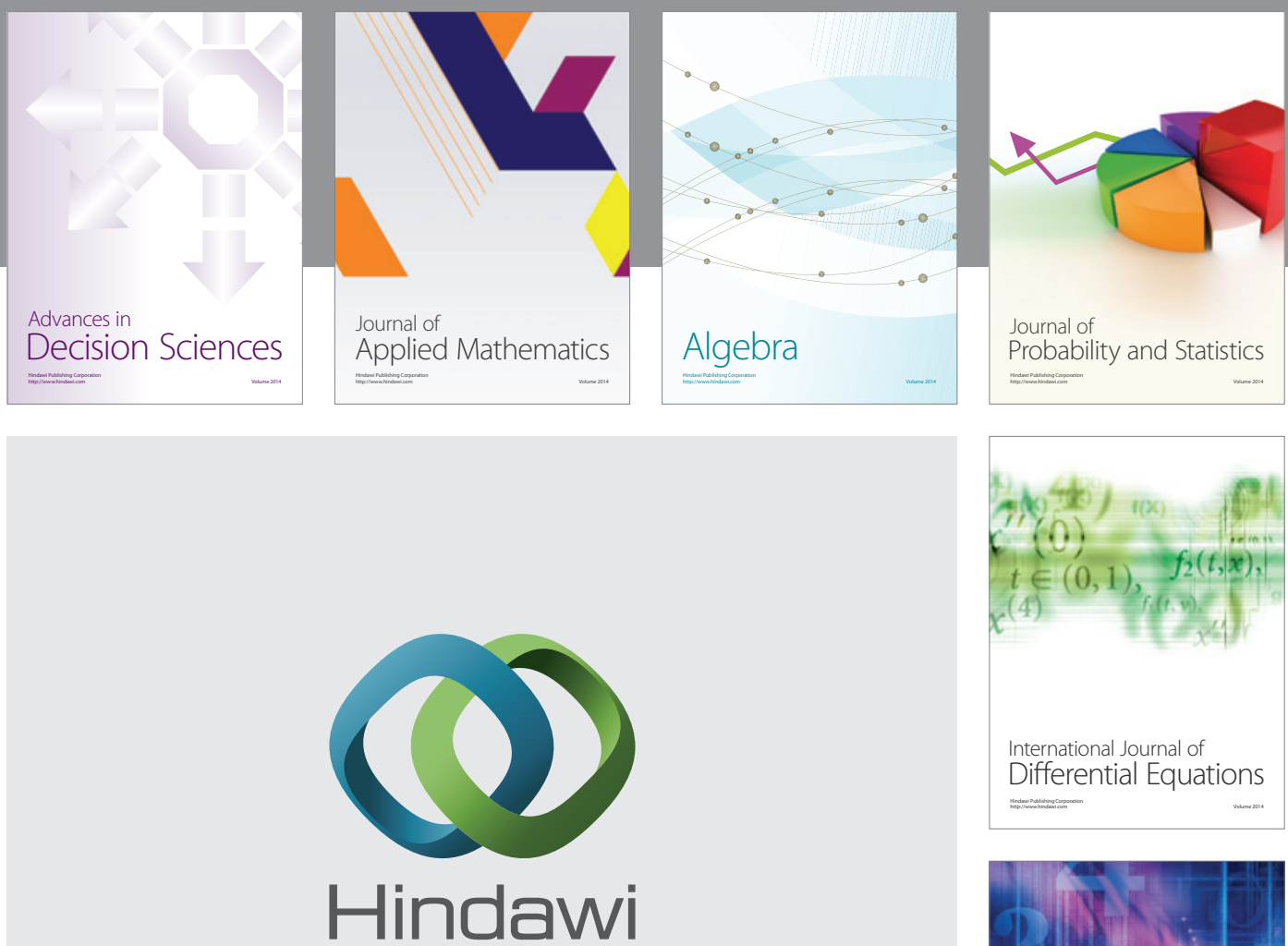

Submit your manuscripts at http://www.hindawi.com
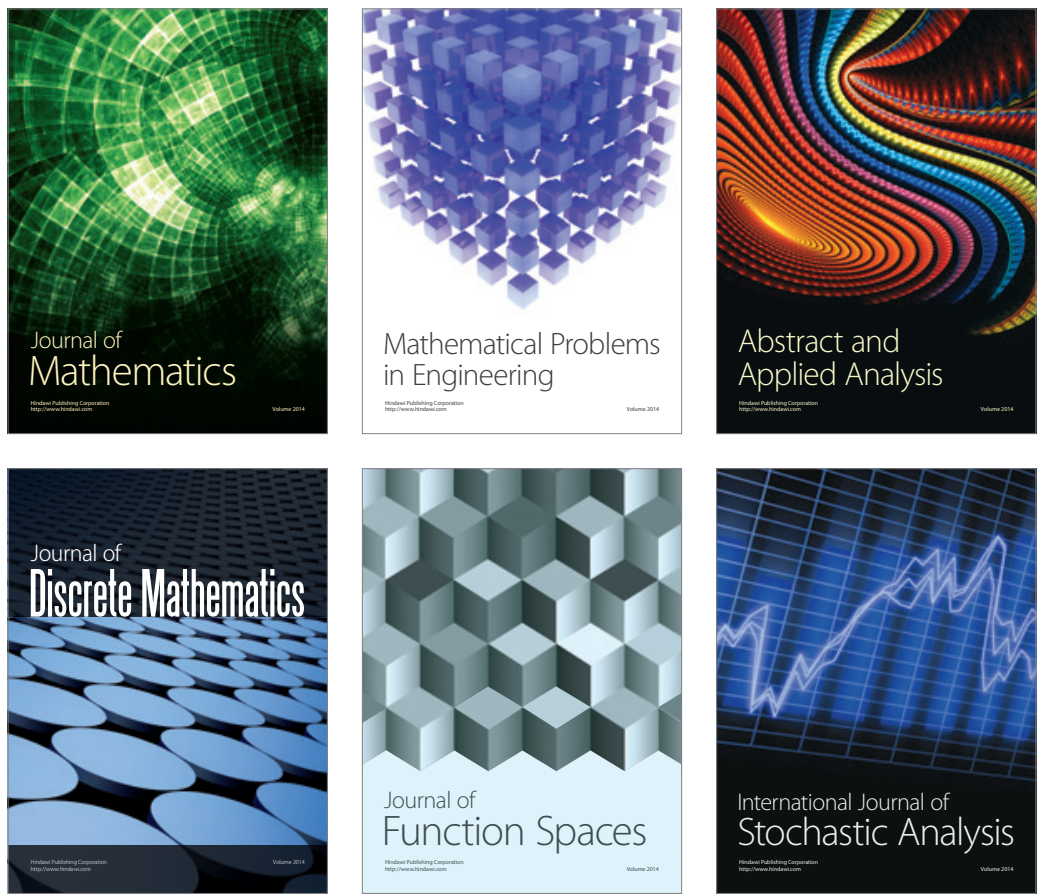

Journal of

Function Spaces

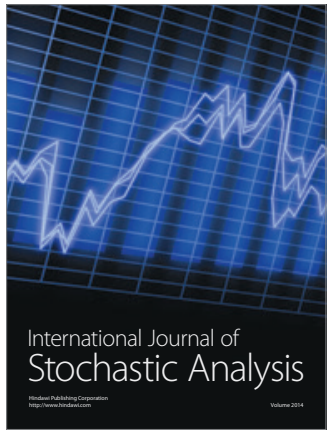

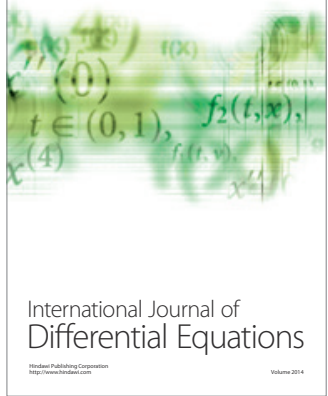
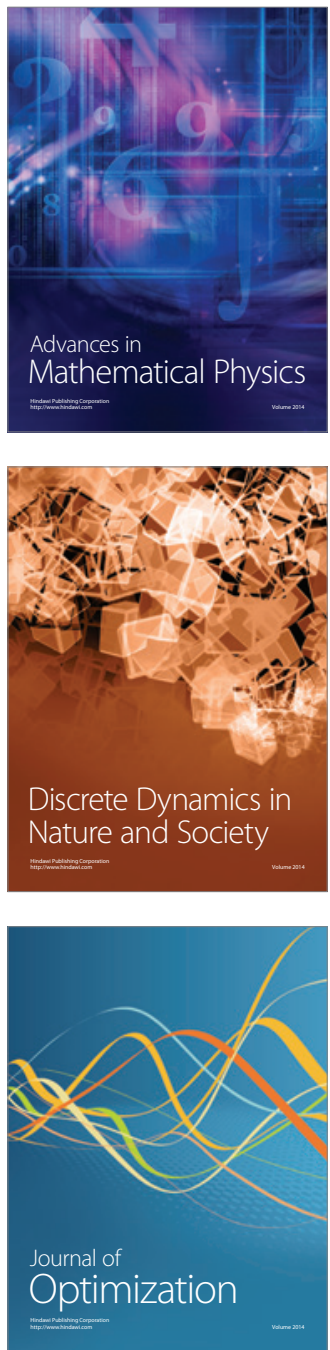